\title{
A man with a spare bladder - large acquired urethral diverticulum
}

\author{
RAY Rupasinghe ${ }^{1}$, S Sudheshan ${ }^{2}$, ND Perera ${ }^{3}$ \\ ${ }^{1}$ Registrar in Surgery, ${ }^{2}$ Senior Urological Registrar, ${ }^{3}$ Consultant Urological Surgeon \\ Department of Urology, The National Hospital of Sri Lanka, Colombo.
}

\section{Case report}

A 44 year-old unmarried male complained of "incontinence" of urine and a small boggy swelling at the base of the penis of 8 years duration. Manual pressure on the swelling resulted in passage of urine per urethra.

He had a road traffic accident (RTA) 12 years ago with pelvic fracture and bleeding per urethra. Pubic bone diastasis was wired and a urethral catheter was passed at the local hospital and kept for 3 months. He had lower urinary tract symptoms after discharge from hospital but it was managed conservatively as the initial (12 years ago) cystourethroscopy was normal.

On examination there was a cystic swelling of 1.5 $\mathrm{cm} \times 1.5 \mathrm{~cm}$ on the ventral aspect of base of penis. About $30 \mathrm{ml}$ of urine could be expressed on manual compression of the swelling.
Ultrasound scan of the abdomen was normal. Uroflowmetry showed an obstructing pattern. Routine urine analysis, microscopy, culture and biochemistry of the blood showed normal values. Urethrogram was reported as a fistulous tract extending from the distal bulbar urethra to superficial perineal pouch (Figure 1). But rigid cystoscopic examination visualised an opening in the penile urethra communicating with the boggy mass at the scrotum confirming the cystic mass a urethral diverticulum (Figure 2).

Diverticulectomy was done and the urethral defect was closed transversely and a Dartos patch was applied over it. He made an uneventful recovery. 


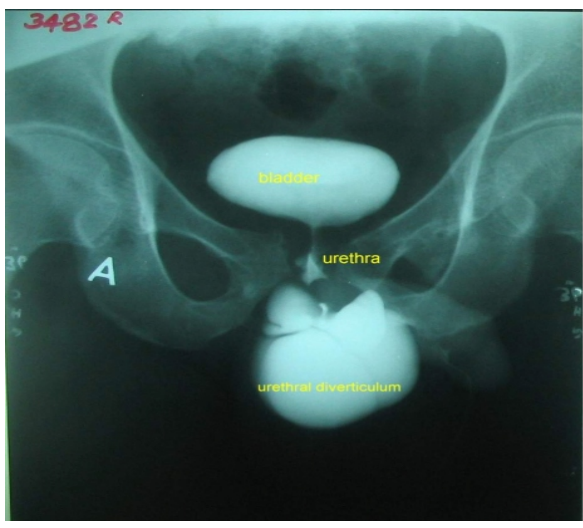

Figure 1 - Urethrogram showing a fistulous connection from the urethra to a superficial perineal pouch

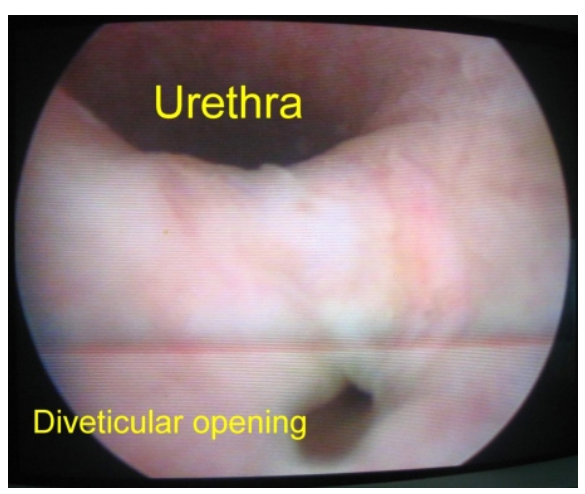

Figure 2 - Rigid cystoscopy showing the fistulous opening from the penile urethra.

\section{Discussion}

Male urethral diverticulae (UD) are rare and have been described mostly in relation to the anterior urethra. More than $90 \%$ are of the acquired variety and result from trauma, instrumentation, surgery, suppuration and urethral calculus or stricture [1].

The common symptoms are irritative lower urinary tract symptoms (LUTS) and recurrent urinary tract infections (UTI). Other complaints include pain, haematuria, obstructive LUTS or urinary retention and incontinence (stress or urge). As in this case, some patients may also have a mass, which upon gentle compression may reveal retained urine or pus discharge through the urethral opening $[1,2]$.

In this patient the urethral stricture which was a result of the previous injury had healed initially, as suggested by the initial cystoscopy and recurred after some time which was not addressed properly. This has resulted in formation of urethral diverticulae, which is a known complication of long standing urethral stricture disease.

Urethrogram usually reveals the diagnosis. Other advanced techniques for the evaluation of UD include double-balloon positive-pressure urethrography (PPU), voiding, cystourethrography (VCUG), ultrasound (US) and magnetic resonance imaging (MRI) with or without an endoluminal coil (eMRI) [3].

Surgical options include either transurethral incision of the diverticular neck or surgical excision. However, all UD do not warrant surgery. Small, asymptomatic once can be left alone. Surgical excision is the treatment of choice but it should be performed with caution. The diverticular sac may be quite attached to the adjacent urethral lumen and careless removal of the sac may result in a large urethral defect requiring construction of a new urethra. Other important considerations during surgery include identification and closure of the diverticular neck and complete removal of the mucosal lining of the diverticular sac to prevent recurrence and a multiple layered closure with Dartous patch to prevent post-operative fistula formation. Endoscopic division of the neck to create a wide communication between the urethra and the diverticulum has also been recommended [4]. If there is an associated urethral stricture, it has to be treated on its own merit. This patient didn't have any significant urethral stricture.

Some patients may have persistence or recurrence of their pre-operative symptoms postoperatively. The finding of a UD following a presumably successful urethral diverticulectomy may occur as a result of a new UD, or alternatively, as a result of recurrence. Recurrence of UD may be due to incomplete removal of the UD, inadequate closure of the urethra or residual dead space or other technical factors. Repeat urethral diverticulectomy surgery can be challenging, as anatomic planes may be difficult to identify [5]. 


\section{Case Reports}

\section{References}

1. Rovner ES: Bladder and Urethral diverticula. In: Campbell-Walsh , Kavoussi LR Novick AC, Partin AW, Peters CA, eds. Campbell's Urology, 9th ed. Philadelphia, W.B. Saunders Co. 2007: 2361-90.

2. Ortilip SA, Gonzalez M, Williams RD: Divericulae of the Male Urethra. J Urol 1980; 124: 350-5.
3. Vijayan P. Ventral urethral diverticulum of fossa navicularis. Indian J Uro1 2006; 22: 148-9.

4. Davis HJ, TeLinde RW: Urethral diverticula: An assay of 121 cases. JUrology 1958: 80: 712-22.

5. Lee RA: Diverticulum of urethra: Clinical presentation, diagnosis and management. Clin Obstet Gynecol 1984: 27: 490-8. 